\title{
WEB 2.0: IS THE ENTERPRISE READY FOR THE ADVENTURE?
}

\author{
Martin Grossman, Bridgewater State College, mgrossman@bridgew.edu \\ Richard V. McCarthy, Quinnipiac University, rmccarthy@quinnipiac.edu
}

\begin{abstract}
The current popularity of social networking is starting to infiltrate the corporate space. Web 2.0 applications, such as blogs and wikis, are increasingly being utilized as ways for businesses to collaborate and share information with employees, customers, partners, and suppliers. Organizations have adopted enterprise architecture approaches to enable them to more quickly react to new technologies. Are organizations ready for Web 2.0? We explain the fundamental concepts in Web 2.0, examine ways it is being utilized in the enterprise, and then analyze if the E2AF enterprise architecture framework is equipped to meet the challenge of Web 2.0
\end{abstract}

Keywords: Enterprise 2.0, Extended Enterprise Architecture Framework, Web 2.0

\section{INTRODUCTION}

One of the biggest stories in 2006 has been the wide scale adoption of social networking and the rise of the next generation of the Web, commonly known as Web 2.0. Time Magazine's recent cover story on this emerging grassroots phenomenon [17] and its bestowal of the Person of the Year designation to 'You,' (meaning the Internet user) is a good indication of how intensely this trend has permeated our culture. The corporate world is also starting to take note, recognizing the potential of social networking as a means to achieve bottom-up knowledge sharing and collaboration. Indeed, the Web 2.0 buzzword has rapidly entered the vernacular of business software vendors, venture capitalists and CIOs alike.

While the enthusiasm surrounding Web 2.0 is in some ways reminiscent of the dot-com craze of the late 90s, there are many in both the academic and practitioner communities who are actively promoting Web 2.0 in the enterprise and who maintain that this time the excitement is not based on 'irrational exuberance', but rather on something much more profound and far-reaching $[6,10]$. At the invitationonly Web 2.0 Summit, recently held in San
Francisco, over 1000 business leaders came together to discuss the future of Web 2.0 and its impact in the corporate environment [6].

Since the current stir surrounding Web 2.0 is prone to so much hype, it is important for IT managers to understand the implications involved with its implementation in the enterprise, and for due diligence prior to plunging ahead full force into this uncharted terrain. This paper provides some background on Web 2.0 applications, technologies and infrastructures and examines the issues surrounding adoption of this new paradigm in the enterprise.

\section{Core Concepts of Web 2.0}

A widely accepted definition of Web 2.0 comes from one of the original innovators behind the concept, Tim O’Reilly of O’Reilly Media, a popular publisher of computer technology materials:

"Web 2.0 is the network as platform, spanning all connected devices; Web 2.0 applications are those that make the most of the intrinsic advantages of that platform: delivering software as a continually-updated service that gets better the more people use it, consuming and remixing data from multiple sources, including individual users, while providing their own data and services in a form that allows remixing by others, creating network effects through an "architecture of participation," and going beyond the page metaphor of Web 1.0 to deliver rich user experiences.” [11]

A closer look at this definition reveals the fundamental concepts inherent in this new model, differentiating it from the first generation of the Web. The concepts of the 'network as a platform' and 'continually-updated service' refer to the fundamental shift in the way software applications are delivered and run, away from the traditional and rigid software adoption cycle, and towards a world of constantly changing web services. Versioning, installations and upgrades are totally transparent to the user. A familiar example would be the carefree way a user might utilize an application like Google, 
totally oblivious to the fact that revisions to the software are being made continuously. In this new environment, the Web functions like a giant computer, and the underlying stack of Internet protocols like a massive 'operating system', working behind the scenes. As users, we are freed from the traditional cycle of designing, developing, testing, shipping and installing packaged software. Rather, applications are available if and when we need them and pieced together in new and creative ways. The term 'Software as a Service' (SaaS), is often used to describe this paradigm. In many ways it is an outgrowth of the Application Service Model (ASP) form of outsourcing that gained in popularity over the last decade.

Another important component of Web 2.0 is the capability of meshing content from disparate sources to form brand new offerings ('consuming and remixing data'). Known as 'mashups', such conglomerations of micro-content leverage data and services from public Web sites and are lightweight in nature, i.e. built with minimal amount of code. They have become increasingly popular across the Web (e.g. Google Maps). The primary business benefit of mashups is that they can be used to quickly meet tactical needs with lower development costs while providing improved user satisfaction. However, the ability to remix data to create new applications is inherently vulnerable, since there is often little control over what is being included from the various Web-based sources.

Perhaps the most compelling aspect of the Web 2.0 proposition is that it allows the average user to play an active role in creating content for the web ('architecture of participation'). Today's youth, the so-called 'Net' generation, seem to have an intuitive understanding of this involvement, as evidenced by the explosive popularity of such social networking environments as MySpace and Facebook. But the concept goes beyond providing teens an outlet to socialize and share favorite photos and MP3s. Whereas Web 1.0 deals with taxonomies, hierarchical classification systems commonly used to organize objective data, the new Web relies on user-driven methods of categorization. Known as 'folksonomies', such classifications are based on user inspired 'tags', freely chosen keywords that are generated by the user community and which allow for less rigid and more intuitive ways of retrieving information. Tagging has become an important part of Web 2.0 sites such as Flickr, Technorati, Delicious, and YouTube, allowing visitors to determine value based on the 'wisdom of the crowd' and not on a top-down authoritative structure.
The participatory architecture of Web 2.0 expands upon the type of collaborative development efforts that previously led to such software mainstays as the Linux operating system and the Apache web server. These software systems became better as the community of enthusiastic contributors revised and improved upon the original. Wikipedia, the collaboratively written encyclopedia, also provides a good example of how such a network effect might take hold. As the number of editors on a particular article increases, the quality of the content theoretically improves. This in turn encourages more individuals to utilize the resource, and so on.

Although it may seem counterintuitive to give away one's internal assets to potential competitors, the open-source model has proven to be an effective strategy for software development and one that is integral to Web 2.0. Many of today's Web 2.0 applications come with open Application Programming Interfaces (APIs), which allow other developers to easily extract from or interact with the application, providing the mashup capability discussed previously.

Another important aspect of today's Web 2.0 environment is that of syndication. Through such XML-based feed formats as RSS (Really Simple Syndication) or Atom, user-specified, continuously updated content may be pushed to the desktop. Today syndication is being used in many new ways to deliver content, such as podcasts from news sites.

\section{THE EMERGENCE OF ENTERPRISE 2.0}

The concepts of knowledge sharing and collaboration did not originate with Web 2.0. Indeed, they are the fundamental ideas behind knowledge management $(\mathrm{KM})$, a discipline that has gained popularity in companies around the world over the last two decades. While KM systems have been around for years, many available solutions are resource intensive, unintuitive and ultimately ineffective [14]. Traditional KM systems are typically top-down, highly centralized and do a poor job of capturing and making visible the tacit knowledge of knowledge workers, which is reflected in their work practices. Such tacit activities, require the making of judgments and the integration of knowledge from exchanges with coworkers, customers, and suppliers, and constitute the bulk of business transactions [2].

Andrew McAfee, a Harvard Business School professor and Web 2.0 evangelist, suggests the term 'Enterprise 2.0' to '”... describe platforms that 
companies can buy or build in order to make visible the practices and outputs of their knowledge workers", and introduces the acronym SLATES to describe the necessary conceptual components [10]. They are:

- Search - to enable users to find what they are looking for.

- Links - to provide users with a guide which indicates what is important and structures online content. The best pages are the ones most frequently linked to.

- Authoring - to allow users to contribute, whether it is knowledge, insight, experience, comments, etc.

- Tags - to let users dictate the way content is categorized

- Extensions - use algorithms to automate some of the categorization and pattern matching (.e.g. Amazon's recommendation system)

- Signals - to alert users when new content of interest appears.

Companies around the world are starting to appreciate the value afforded by Web 2.0 in the enterprise and case studies have started to appear in the literature [5, 7, 12, 14, 15, 17]. Among the benefits attributed to blogs and wikis in the enterprise are: (1) they provide an inexpensive way to gain web presence and (2) they provide a mechanism by which companies can stay in touch with customers for disseminating product information and for obtaining feedback, (3) they are relatively easy to set up [18].

Well-known strategist Don Tapscott, in his new book entitled Wikinomics, [16], claims that Web 2.0 has already propelled the business world into a new era, one of mass collaboration. New business models are emerging that challenge traditional business designs. They are: (1) Peer Pioneers - the shift to peer-to-peer networks and the open-source movement, (2) Ideagoras - the ability to tap global pools of highly skilled talent for ideas, inventions, virtual collaboration., (3) Prosumers - dynamic world of customer innovation and the intentional 'hackability' of Web services, (4) New Alexandrians - the new science of sharing that will accelerate scientific discovery and that will ultimately help the world address its most difficult problems (e.g. environment, human health), (5) Platforms for Participation - how companies are moving from proprietary formats to platforms that are open and that encourage communities of partners to create value, (6) Global Plant Floor - manufacturing- intensive industries are moving towards global ecosystems for design and development of goods, and finally, (7) Wiki Workplace - how mass collaboration is taking hold in the business world, creating a new organizational structures based on merit, and tearing down traditional hierarchical structures.

In spite of the excitement that Web 2.0 is generating among industry pundits, it is important for IT managers to carefully consider the ramifications of adopting such platforms. There are some significant barriers, both technical and cultural, which need to be addressed. On the technical side, security is perhaps the biggest concern. Issues involving securing sensitive information behind the firewall, controlling access to levels of information and databases, and protecting the integrity of information from tampering by disgruntled employees, are all commonly cited issues [3, 9].

Cultural barriers are no less important. McAffee [10] highlights the following Enterprise 2.0 best practices (1) create a receptive culture to prepare the way for new practices (2) create a common platform to allow for a collaboration infrastructure, (3) use an informal rollout approach as opposed to a more formal procedural one, and (4) get managerial buy-in. An important consideration in implementing Web 2.0 is the loss of control that managers may perceive as their systems are opened up. As McAfee [10] states:

“These tools may well reduce management's ability to exert unilateral control and to express some level of negativity. Whether a company's leaders really want this to happen and will be able to resist the temptation to silence dissent is an open question. Leaders will have to play a delicate role if they want Enterprise 2.0 technologies to succeed.”

\section{UNDERLYING TECHNOLOGIES AND ARCHITECTURAL ISSUES}

A number of key technologies provide the functionality behind Web 2.0 applications. One is Ajax (Asynchronous JavaScript and XML), a lightweight scripting technique that is changing the face of the web. While still very new, Ajax has become a very popular approach to providing rich, desktop-like content to users. The power of Ajax can be appreciated by viewing such applications as Google Maps and Netflix, which are considered to be Rich Internet Applications (RIA). Ajax improves upon the traditional page-oriented approach to 
delivering web content. It is built upon the HyperText Markup Language (HTML), and has evolved from Dynamic HTML (DHTML), which incorporates other technologies such as JavaScript and Cascading Style Sheets (CSS) with HTML to provide rich user content.

Users today are no longer satisfied with simple browser-based displays of text and graphics information. Ajax provides a new environment that enables web pages to behave more like desktop applications, in that they do not need to be reloaded from the server on each user input. Instead of requiring repeated page refreshes, Ajax provides a mechanism for small amounts of data to be exchanged with the server, in the background while the user is still fully engaged in the application. Such incremental updates are made possible via builtin functions provided by JavaScript and the XMLHttpRequest API, both integral components of Ajax.

While it is easy to see how Web 2.0 technologies, such as Ajax, can add enriched content and value to the business user, it is important to consider the accompanying risks. Enterprise wide implementation of such technologies brings with it fundamental changes to the IT infrastructure and enterprise architecture [9]. Hogging of precious, mission critical systems resources may indeed be too high a price to pay for many harried IT managers desperately trying to maximize performance and return on investment.

The infrastructure underlying Web 2.0 did not appear in a vacuum; it has evolved out of other, more stable platforms. SOAP (Simple Object Access Protocol), for example, has been the primary protocol used for Web Services over the last decade. It has reached a level of maturity, is well understood and reliable. However, many businesses rushing to implement Web 2.0 are bypassing SOAP and using a newer, less robust model, called REST (Representational State Transfer). While REST is easier to implement than SOAP, it is also less secure, scalable, and maintainable.

The lightweight nature of Web 2.0 technologies, such as Ajax, REST and mashups, may create additional pressure on already heavily taxed back-end servers in the enterprise. Many IT managers are not considering the extra requirements that become necessary in terms of monitoring, management, deployment of this pieced together architecture and the implications that Web 2.0 implementation has in the enterprise [9].
Another potential concern is the lack of interoperability afforded by Web 2.0 platforms. For example, it is extremely difficult to migrate from one Ajax toolkit to another. In spite of recent efforts by the OpenAJAX Alliance to define a single declarative markup language for developers, the landscape is still a free for all, with little interoperability.

Other than Web services standards, such as SOAP, WSDL (Web Services Description Language), and UDDI (Universal Description, Discovery, and Integration), there are no widely accepted standards on which vendors can base new Web 2.0 products. Standards groups, such as W3C (World Wide Web Consortium) and OASIS (Organization for the Advancement of Structured Information Standards) have yet to make a significant impact on promoting new standards in this area.

\section{THE ENTERPRISE 2.0 MARKET}

The lack of robust enterprise grade security and standards infrastructures notwithstanding, vendors have rushed into the Web 2.0 space, offering a plethora of new products. Companies such as Nexaweb (www.nexaweb.com), JackBe (www.jackbe.com) and ActiveGrid (www.activegrid.com), for example, have established themselves as first movers in the Enterprise 2.0 systems development space.

Many business software vendors are also incorporating Web 2.0 ideas, such as blogging and file sharing, into their offerings. iUpload's Customer Conversation System (http://www.iupload.com), for example, incorporates corporate blogging and wiki platforms, and also includes security, workflow and regulatory compliance tools. Tacit Software's Illumio (http://www.ilumio.com) is a web-based information broker that matches end user information requests with users in the company who might know the answer. Koral (www.koral.com) is a web-based document collaboration and sharing tool which also categorizes documents automatically and notifies users of updates and new documents published by authors or topics to which they have subscribed. [14].

Web 2.0 has also entered the radar screens of the big players in the industry, not just the stereotypical startup companies. IBM has been experimenting with Web 2.0 development tools and has recently announced its Enterprise Mashup platform, which is currently being test-driven with selected customers $[1,8]$. The new toolset, which is dependent on Ajax technology, is said to blend external information and 
web services such as news feeds, weather reports, etc., with enterprise content. Such 'mashups' can help meet specific business needs with relative speed.

Even Microsoft, the preeminent vendor in the traditional client/server world, is taking the Enterprise 2.0 phenomenon seriously and starting to develop strategies to adapt to the changes on the horizon. Seeing a vast market for tools to help tame the wilds of this new landscape, the company has embraced a strategy to provide a new suite of management tools, hoping it can carve out a significant niche in the Enterprise 2.0 market. [19].

\section{EXTENDED ENTERPRISE ARCHITECTURE FRAMEWORK}

The Extended Enterprise Architecture Framework was developed by the Institute for enterprise architecture developments in 2002 as an extension of the Zachman framework, the Enterprise Architecture Planning Framework and the Federal Enterprise Architecture Framework. It takes a holistic approach to enterprise architecture addressing the four common enterprise architecture platform components: business workflow, information management, technical infrastructure and applications by utilizing a streamlined set of views that describe the areas of concern for information technology within an organization. It emphasizes communication across all stakeholders in an organization to provide flexibility and to adapt to changing business environments [13].

There are six levels of concern which interest with the business, information, information systems and technology infrastructure perspectives creating a matrix grid. The six levels of concern include:

- $\quad$ The Contextual level describing the vision, strategy, principles and environmental context of the organization. It provides an extended context of the organization and the scope of the enterprise architecture styled describing both the business and technical drivers that an organization requires.

- $\quad$ The Environmental level describing the formal extended business relations and the related information flows. The extended enterprise interoperability is described. The business and technology relationships within the extended enterprise are represented. This defines points of collaboration and the structure of governance within the extended enterprise.
- $\quad$ The Conceptual level addresses the requirements representation. The business requirements and relationships are documented, Functional specifications are documented to provide a comprehensive view of the each business function and how each technical function support those goals.

- $\quad$ The Logical level describes the types of collaborations that take place within a business process. Product independent reference solutions (PIRS) are addressed to show the logical solutions within each aspect area.

- $\quad$ The Physical level addresses solution representation. Product specific reference solutions are documented and technical reference models show physical solutions including business and communication changes, supporting software products and tools, hardware and communication products.

- $\quad$ The Transformational level describes the impact to the organization of the proposed solutions. The transformational roadmap that supports each business area is described.

\section{CONCLUSION}

The second generation of the Web has arrived and it is enabling new ways for companies to reach their customers, build relationships and promote their brands. Applications like blogs, wikis, podcasts, and mashups are rapidly making there way into the enterprise.

However, Web 2.0 technologies are still evolving and the industry is immature. Issues such as security and interoperability are still very real concerns. There are also a number of important challenges managers need to consider, such as monitoring the output of individuals to ensure that it reflects company policy, legal issues related to the type of information that is being shared, and distinguishing between timewasting socializing on the web and productive work.

In spite of the barriers, companies large and small are jumping onto the Web 2.0 bandwagon, and exploring ways to leverage social networking and collaboration as competitive strategies. New products are entering the market daily. To be fair, not everyone is gungho about Web 2.0. Tim Berners-Lee, credited with inventing the World Wide Web, has expressed his annoyance with the whole phenomenon, dismissing it as useless jargon that nobody can explain and a technology that is not significantly different than Web 1.0 [4]. 
Time will tell if the whole Enterprise 2.0 phenomenon blows over and becomes yet another IT silver bullet. At the present time, however, the excitement continues and there does not seem to be any waning of interest in this new paradigm for webbased applications.

Organizations that have invested in enterprise architecture anticipate it will position them to flexibly incorporate new technology that is of value. The myriad of enterprise architecture frameworks support this to varying degrees. The Extended Enterprise Architecture Framework provides a flexible framework that appears to be well suited to easily allow for the incorporation of Web 2.0 technologies into an existing web infrastructure. It emphasizes establishing communication structures within an organization to rapidly respond to changing business needs and takes into account the differing relationships amongst stakeholders in the organization. We propose to empirically test if users of this framework perceive it to be of value and support Web 2.0 technologies.

\section{REFERENCES}

1. Baer, T. (2006). IBM pushes enterprise mashups. Computer Business Review Online. June 16, 2006. Available at :

http://www.cbronline.com/article_news_print.as p?guid=8AC9900E-9993-4E5B-A4B59282115D918A

2. Beardsley, S., Johnson, B. and Manyika, J. M. (2006). Competitive advantage from better interaction. McKinsey Quarterly. June 1, 2006, Issue 2.

3. Boyles, J. (2006). Top 10 Management Fears About Enterprise Web 2.0. Enterprise Web 2.0. July 11, 2006. Available : http://www.enterpriseweb2.com/?p=10

4. Clarke, G. (2006). Berners-Lee calls for Web 2.0 calm. The Register. Available at: http://www.theregister.com/2006/08/30/web_20_ berners_lee/

5. Dennison, R. (2006, November/December). Using wikis for collaboration and KM at BT. KM Review. 9(5).

6. Hinchcliffe (2006). The State of Web 2.0. Web Services Journal. April 4, 2006. Available at http://web2.wsj2.com/the state_of_web_20.htm

7. Hof, R. (2006). Web 2.0 has corporate America spinning. BusinessWeekOnline. July 5, 2006.

Available at

http://www.businessweek.com/technology/conte nt/jun2006/tc20060605_424102.htm
8. IBM (2006). IBM executive declares Web 2.0 technology to drive new business applications. Available at http://www03.ibm.com/press/us/en/pressrelease/19821.wss.

9. MacVittie, L.(2006). Danger 2.0.: Is one of the most hyped technologies in our industry too untamed for the enterprise? Network Computing. October, 26, 2006. 40-54.

10. McAfee, A. P. (2006). Enterprise 2.0: The dawn of emergent collaboration. MIT Sloan Management Review, 21-28.

11. O’Reilly, T. (2005). Web 2.0: Compact Definition? Available at: http://radar.oreilly.com/archives/2005/10/web_2 0_compact_definition.html.

12. Scarff, A. (2006, September/October). Advancing knowledge sharing with Intranet 2.0: How IBM, Motorola and Siemens are using blogs, wikis and RSS. KM Review. 9 (4). 24-27.

13. Schekkerman, J. (2006). Extended Enterprise Architecture Framework (E2AF) Essentials Guide. Available at http://www.enterprisearchitecture.info/Images/E2AF/Extended\%20Ent erprise\%20Architecture\%20Framework\%20Esse ntials\%20Guide\%20v1.5.pdf

14. Spanbauer, S. (2006). Knowledge Management 2.0. CIO Magazine. December 1, 2006.

Available at: : http://www.cio.com/archive/120106/fea_tec.html

15. Sternstein, A. (2006). Web 2.0 for Feds. Federal Computer Week. November 20, 2006. 30-34.

16. Tapscott, D. and Williams, A. D. (2006). Wikinomics: How Mass Collaboration Changes Everything. Penguin Group. New York, N.Y.

17. Time (2006). Person of the Year. Time Magazine. December 25, 2006- January 1, 2007. 38-76.

18. Wood, W., Behling, R. and Haugen, S. (2006). Blogs and business: Opportunities and headaches. Issues in Information Systems. 7(2). 312- 316.

19. Worthen, B. (2006). Beyond Vista: Inside Microsoft's plan to dominate the Web 2.0 enterprise. CIO Magazine, November 15, 2006. 